\title{
Fungal arabinan and L-arabinose metabolism
}

\author{
Bernhard Seiboth • Benjamin Metz
}

Received: 9 October 2010 /Revised: 8 December 2010 /Accepted: 8 December 2010 / Published online: 7 January 2011

(C) The Author(s) 2011. This article is published with open access at Springerlink.com

\begin{abstract}
L-Arabinose is the second most abundant pentose beside D-xylose and is found in the plant polysaccharides, hemicellulose and pectin. The need to find renewable carbon and energy sources has accelerated research to investigate the potential of L-arabinose for the development and production of biofuels and other bioproducts. Fungi produce a number of extracellular arabinanases, including $\alpha$-L-arabinofuranosidases and endo-arabinanases, to specifically release L-arabinose from the plant polymers. Following uptake of L-arabinose, its intracellular catabolism follows a four-step alternating reduction and oxidation path, which is concluded by a phosphorylation, resulting in D-xylulose 5-phosphate, an intermediate of the pentose phosphate pathway. The genes and encoding enzymes Larabinose reductase, L-arabinitol dehydrogenase, L-xylulose reductase, xylitol dehydrogenase, and xylulokinase of this pathway were mainly characterized in the two biotechnological important fungi Aspergillus niger and Trichoderma reesei. Analysis of the components of the L-arabinose
\end{abstract}

B. Seiboth · B. Metz

Research Area Gene Technology and Applied Biochemistry, Institute of Chemical Engineering,

Vienna University of Technology,

Wien, Austria

B. Seiboth $\cdot$ B. Metz

ACIB GmbH c/o Institute of Chemical Engineering,

Vienna University of Technology,

Wien, Austria

B. Seiboth $(\square)$

Molecular Biotechnology Group, Research Area Gene

Technology and Applied Biochemistry, Institute of Chemical

Engineering, Vienna University of Technology,

Gumpendorferstrasse 1a, 166-5, 1060 Wien, Austria

e-mail: bseiboth@mail.tuwien.ac.at

URL: http://www.vt.tuwien.ac.at/ pathway revealed a number of specific adaptations in the enzymatic and regulatory machinery towards the utilization of L-arabinose. Further genetic and biochemical analysis provided evidence that $\mathrm{L}$-arabinose and the interconnected D-xylose pathway are also involved in the oxidoreductive degradation of the hexose D-galactose.

Keywords Trichoderma reesei $\cdot$ Aspergillus niger . $\alpha$-L-arabinofuranosidase $\cdot \mathrm{D}$-xylose $\cdot \mathrm{D}$-galactose

\section{Introduction}

The monosaccharide L-arabinose is an aldopentose and the second most abundant pentose beside D-xylose in plants. Although most monosaccharides are normally present in nature in their D-form, L-arabinose is a rare exception to this rule and found mainly in its furanose form as a component of the plant biopolymers hemicellulose and pectin. Studies on the catabolic pentose pathway, which includes beside Larabinose the interconnected D-xylose catabolism has received less attention in previous years. One reason might be that both pathways are not present in the conventional Saccharomyces cerevisiae laboratory strains strain, and only recently, D-xylose fermenting wild-type isolates were described (Wenger et al. 2010). However, due to the increased interest in conversion of plant biomass to biofuels/ethanol and other bioproducts, such as xylitol, etc, the catabolism of these pentoses has now received a strong attention. A fungal D-xylose catabolic pathway was established much earlier, which might be due to the fact that the lignocellulosic food stocks contain, on average, much less L-arabinose than D-xylose (e.g., corn stover contains $19 \%$ xylan and 3\% arabinan, wheat bran contains $19 \%$ xylan, and $15 \%$ arabinan (Hayn et al. 1993)), and that 
the L-arabinose path needs five enzymes, while D-xylose only three enzymes for the conversion to D-xylulose 5phosphate (Fig. 2). Both fungal D-xylose and L-arabinose pathways were introduced into $S$. cerevisiae strains to improve conversion of these pentoses to ethanol (for a review: Hahn-Hägerdal et al. 2007). In addition, numerous new arabinan degrading enzymes were characterized within the last years and can now be used to supplement available enzyme mixtures for a faster and more complete conversion of L-arabinose and arabinan containing feed stocks. This mini review focuses on fungi, with particular emphasis on A. niger and T. reesei (syn. Hypocrea jecorina) but will exclude most of the available data on yeasts. After a short survey on the extracellular arabinanolytic degradation system, special attention will be paid to the elucidation of the components of the catabolic L-arabinose pathway, their role in D-galactose utilization, and the regulation of the arabinanolytic pathway.

\section{Degradation of arabinan and L-arabinofuranose containing plant polysaccharides}

In the plant cell wall, L-arabinose is present in arabinoxylan, arabinogalactan, and arabinan. Due to the diversity of the structures, different hydrolases are needed for the release of L-arabinose residues from these polymers. The complete degradation of hemicellulose and pectin requires the concerted action of many different arabinanolytic enzymes (Fig. 1). Endo-processive arabinanases (Arabinan endo-1,5- $\alpha$-L-arabinosidase; EC 3.2.1.99) cut randomly within the backbone of the arabinan, while exo-acting $\alpha$-Larabinofuranosidases (ABF, $\alpha-\mathrm{N}$-arabinofuranosidase, $\mathrm{EC}$
3.2.1.55) release terminal non-reducing $\alpha$-L-arabinofuranoside residues from different $\alpha$-L-arabinosides, arabinans, arabinoxylans, and arabinogalactans. In addition, arabinoxylan arabinofuranohydrolases (AXH) specifically remove arabinofuranose side chains that are linked to backbone D-xylose residues and exo- $\alpha-1,5$-L-arabinanases release L-arabinose or short arabino-oligosaccharides. $\alpha$-LArabinofuranoside activities are also found in a group of $\beta$-xylosidases, and some xylanases are also involved in arabinan degradation (de Vries and Visser 2001). All these enzymes, their genomic, structural and biochemical information are found in carbohydrate-active enzyme database (CAZy; http://www.cazy.org/), which divides the structural-related catalytic modules of the enzymes in different glycoside hydrolase (GH) families (Cantarel et al. 2009). In the following, only a general overview will be given. Their biotechnological applications, including improvement of wine flavors, pulp treatment, juice clarification, production of important medicinal compounds, and bioethanol, was reviewed elsewhere (e.g., Numan and Bhosle 2006; Saha 2000; Shallom and Shoham 2003).

The best studied group and by far the most complex one are the ABFs. They belong to the catalysts with highest efficiency known (Rye and Withers 2000; Shallom et al. 2002). ABFs are grouped mainly in four families GH43, GH51, GH54, and GH62 (Cantarel et al. 2009) and hydrolyse $(1 \rightarrow 2),(1 \rightarrow 3), \quad(1 \rightarrow 5) \quad \alpha$-L-arabinofuranosyl linkages. $\mathrm{ABFs}$ were previously classified according to their substrate specificity in (1) ABFAs (GH51) which are not active towards arabinan, (2) in polymer hydrolyzing ABFBs (GH54, GH43), and (3) AXHs specific for arabinoxylans (Beldman et al. 1997). However, since the
Fig. 1 Degradation of different L-arabinose polymers in pectin. Different types of arabinan and arabinogalactan are present as side chains of rhamnogalacturonan I in pectin. Endoprocessive arabinanases $(\mathrm{ABN})$ attack randomly the backbone of arabinan, while exo-acting $\alpha$-L-arabinofuranosidases (ABF) release the terminal $\alpha$-L-arabinofuranoside residues

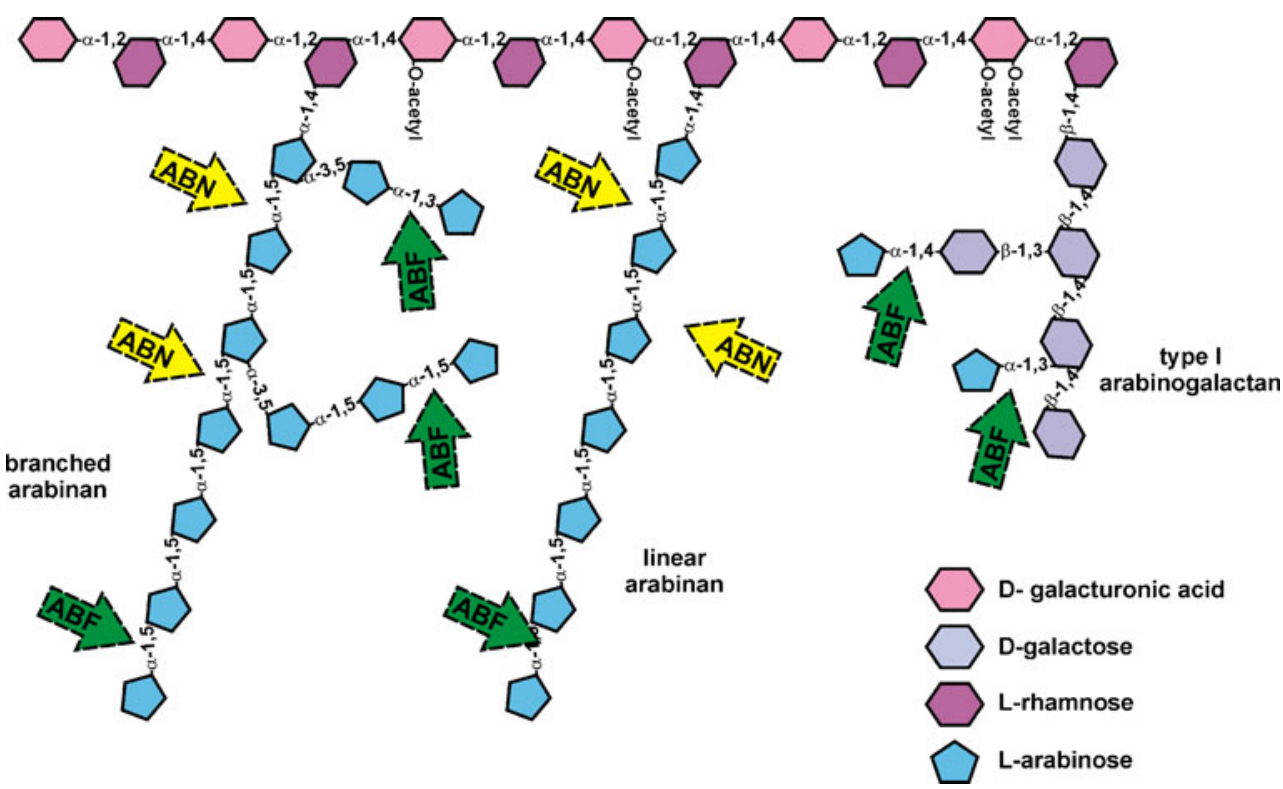


substrate specificity differs within a single GH family, the CAZy classification is now generally accepted (Saha 2000). ABFs hydrolyze glycosidic bonds via acid/base-assisted catalysis, which can lead either to retention (GH3, GH51 and GH54) or inversion (GH43) of the anomeric configuration (Zechel and Withers 2000; de Groot et al. 2003; Numan and Bhosle 2006). GH54 enzymes have a specifically adapted two domain structure, which includes a catalytic and a substrate binding domain. This carbohydrate-binding module (CBM42 family) is specific for monomeric L-arabinose residues of polysaccharides. This binding side plays an effective role in the degradation of insoluble pectin and arabinoxylan, as mutations in the CBM42 resulted in a decreased activity (Miyanaga et al. 2006). The close binding to L-arabinose residues and its hydrolysis may be the result of a fine-tuning link from arabino-oligosaccharides to cellulose degradation where the cellulose microfibrils are in close interaction with hemicelluloses, containing L-arabinose residues (Zykwinska et al. 2005). Recent studies revealed that the genome of Penicillium funiculosum contains four GH54 ABF with three conventional GH54 ABF, while in one ABF, CBM42 is replaced by a 100 aa shorter cellulose-binding domain CBM1 (Guais et al. 2010).

Endo-arabinanases belong to the GH43 family, which contains also $\mathrm{ABFs}$ and $\beta$-xylosidases. Endo-arabinanases are the only enzymes hydrolysing $(1 \rightarrow 5) \alpha$-L-arabinofuranosyl bonds within the arabinan polysaccharide. At present, only one type of endo-arabinanase gene was characterized in different Aspergilli spp. (Flipphi et al. 1993; de Vries and Visser 2001; Skjot et al. 2001). Also, reports on exoarabinanase from GH93 family are rare and they are found, e.g., in Penicillium chrysogenum (Sakamoto, 2004) and in Fusarium graminearum. The latter displayed a strict substrate specificity for linear $\alpha-1,5-1$-arabinan and releases arabinobiose from the nonreducing end (Carapito et al. 2009).

AXHs from GH62 family specifically cleave $(1 \rightarrow 2)$ or $(1 \rightarrow 3) \alpha$-L-arabinofuranoside residues only from xylans. GH62 family AXHs were characterized from A. niger and A. thubingiensis (Gielkens et al. 1997), while ABFs from the GH62 family were characterized in, e.g., A. sojae. Whether there is a real difference within GH62 between $\mathrm{AXH}$ and these $\mathrm{ABF}$ needs further clarification as for the A. sojae GH62 enzyme, which shows a high sequence identity to the AXHs of A. niger and A. thubingiensis, the substrate specifity was observed to be different as the enzyme acted not only on arabinoxylan, but also on arabinan and arabinogalactan (Kimura et al. 2000).

$\beta$-xylosidases with $\mathrm{ABF}$ activity are found in GH3 family. Studies in A. niger and T. reesei showed that $x \ln D /$ bxll encodes for the major extracellular $\beta$-xylosidase responsible for the liberation of D-xylose from D-xylose polymers. Experiments indicate that the $\beta$-xylosidase has a further $\mathrm{ABF}$ activity, which could only be observed with synthetic $p$-nitrophenyl- $\alpha$-arabinofuranoside as substrate but not with, e.g., arabinoxylan (Herrmann et al. 1997, van Peji et al. 1997, Margolles-Clark et al. 1996).

Although arabinan degrading enzymes are present in most fungi, a number of fungi seem to miss endoarabinanases (e.g., T. reesei, Akel et al. 2009), which explains the reduced growth on arabinan polysaccharide and might limit their ability to attack intact plant cell walls.

\section{The L-arabinose catabolic pathway}

For the microbial degradation of L-arabinose, a number of different pathways were described. The best studied prokaryotic pathway includes an isomerase step (Schleif 2000) where L-arabinose is converted to D-xylulose 5-phosphate by L-arabinose isomerase, L-ribulokinase, and L-ribulose-5phosphate 4-epimerase. In addition, prokaryotic oxidative pathways were described (Watanabe et al. 2006; Dahms and Anderson 1969), which result in either the TCA cycle metabolite $\alpha$-ketoglutarate or pyruvate and glycolaldehyde. Fungi have a unique oxidoreductive pathway, which produces, in analogy to the bacterial isomerase pathway, Dxylulose 5-phosphate. The basic biochemistry of L-arabinose catabolism was established in P. chrysogenum (Chiang and Knight 1961) and latter in A. niger (Witteveen et al. 1989). The pathway is characterized by four alternating reductionoxidation reactions (Fig. 2), which convert L-arabinose via Larabinitol (NADPH), L-xylulose $\left(\mathrm{NAD}^{+}\right)$and xylitol (NADPH) to D-xylulose ( $\mathrm{NAD}^{+}$dependent). In a final irreversible step, D-xylulose is phosphorylated to D-xylulose 5-phosphate, a substrate of the pentose phosphate pathway. These steps are catalyzed in the following order by Larabinose reductase, L-arabinitol dehydrogenase, L-xylulose reductase, xylitol dehydrogenase, and xylulokinase. Although this process is overall redox neutral, it results in an imbalance of the redox cofactors since reductases use $\mathrm{NADPH}$ while dehydrogenases $\mathrm{NAD}^{+}$as cofactor. Within the last years, the corresponding genes and proteins were characterized, which provides now an (almost) complete picture of this fungal-specific route. The other abundant pentose D-xylose is catabolized in a similar way, and both pathways are interconnected with xylitol as the first common metabolite of the shared part (Fig. 2). Genes, encoding the enzymes for these pathway, are present in most fungal genomes, in some cases, an amplification of some of the pathway genes has taken place, leading to a number of paralogous genes (Flipphi et al. 2009). The analysis of the pathway is further complicated by the presence of two or even more enzymes with similar substrate specificities, which can compensate for the loss of a specific pathway gene to varying degrees. Although these enzymes are not 
Fig. 2 L-arabinose and D-xylose catabolism in fungi. The first step of the L-arabinose pathway is catalyzed by two different aldose reductases. In $A$. niger, the L-arabinose-specific reductase (LarA) is responsible for the main activity, while in $T$. reesei the D-xylose reductase XYL1 is responsible for the first step in L-arabinose assimilation. The last two shared steps of the two pentose catabolic pathways result in D-xylulose 5-phosphate, which is further assimilated via the pentose phosphate pathway

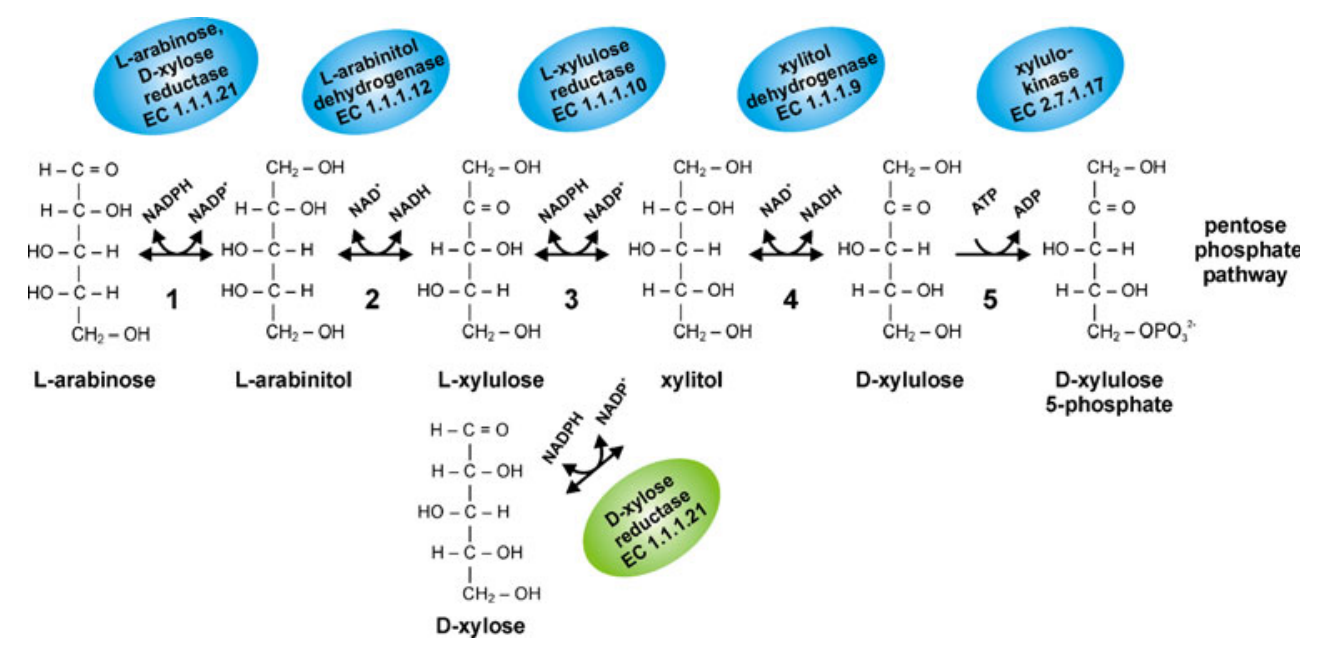

directly involved in L-arabinose catabolism but, e.g., in Dxylose catabolism, they are present during normal growth on L-arabinose or specifically expressed in the absence of one of the L-arabinose pathway enzymes.

The nature of the enzyme(s) catalyzing the first NADPH-dependent reduction of L-arabinose differs depending on the organism. This step is catalyzed by members of the aldo-keto reductase family, and deletion analysis of $T$. reesei and $A$. niger suggests that at least two enzymes are involved in this step. In $T$. reesei, the main reductase is the $\mathrm{D}$-xylose reductase (XYL1), which accounts for most of the reductase activity on both Larabinose and D-xylose. Deletion of the $T$. reesei $x y l 1$ results in significantly impaired growth on both sugars (Akel et al. 2009; Seiboth et al. 2007). In A. niger, the situation is different: a reductase with preferred specificity for L-arabinose has been described (de Groot et al. 2005), and recently, the corresponding gene $\operatorname{lar} A$ was cloned (Mojzita et al. 2010a). LarA is induced by L-arabinose, and the recombinant-produced enzyme converts L-arabinose and also D-xylose to their corresponding sugar polyols with a higher affinity for L-arabinose. Growth on Larabinose as carbon source and L-arabinose reductase activity are decreased by a $\operatorname{lar} A$ gene knockout. A candidate for the remaining L-arabinose reductase activity is the Dxylose reductase XyrA, an orthologue of the $T$. reesei XYL1, which shows overlapping substrate specificity with the L-arabinose reductase (de Groot et al. 2005).

An L-arabinitol 4-dehydrogenase was first cloned in $T$. reesei (Richard et al. 2001). Deletion of lad1 leads to a loss of growth on L-arabinose and L-arabinitol and the effect is, therefore, similar to the araA1 mutation in A. nidulans (de Vries et al. 1994; Pail et al. 2004). Genetic evidence further showed that the $T$. reesei LAD1 can partially compensate for the loss of the xylitol dehydrogenase step in an $x d h 1$ negative strain during growth on D-xylose and xylitol (Seiboth et al. 2003).
The cloning of the fungal L-xylulose reductase (LXR) proved to be difficult, and still, more research efforts are needed to fully comprehend this step. The first gene encoding a fungal enzyme with L-xylulose reductase activity was identified by a genetic screening of genes, which enabled growth on L-arabinose of a $S$. cerevisiae strain that expressed all genes necessary for L-arabinose catabolism with the exception of LXR (Richard et al. 2002). However, deletion of $l x r l$ in $T$. reesei did not affect growth on L-arabinose/L-arabinitol or the L-xylulose reductase activity in cell extracts of $\mathrm{L}$-arabinose grown $T$. reesei. Based on aa sequence similarities and a reduction of $\mathrm{D}$ mannitol dehydrogenase activity in a $l x r l$ deletion strain, we concluded that the encoded protein is actually a Dmannitol dehydrogenase involved in developmental processes (Metz et al. 2009). Attempts to clone the 'true' NADPH-dependent L-xylulose reductase resulted in the Larabinose inducible $A$. niger $\operatorname{lx} A$. The $A$. niger LxrA shows a much higher specific activity (450 U/mg compared to 4.8 U/mg) than the $T$. reesei LXR1 and is more specific for L-xylulose than LXR1, which shows also activity against some other ketose sugars, including D-xylulose, D-fructose, and L-sorbose (Richard et al. 2002). LxrA deletion results in strains lacking almost all NADPH-specific L-xylulose reductase activity. Surprisingly, only a slight reduction of growth on L-arabinose was observed. A useful hint towards the further fate of L-xylulose seems to be that NADHdependent activities are present in the deletion strain, which were even slightly increased (Mojzita et al. 2010b). In $A$. niger, both L-xylulose reductase activities had been described before and are induced by L-arabinose and to a lesser extent also by D-xylose (Witteveen et al. 1989). A candidate for such an activity could be orthologues of the NADH-dependent L-xylulose reductase Alx1 of the yeast Ambrosiozyma monospora (Verho et al. 2004), but their relevance for the fungal L-xylulose step still needs to be established. Interestingly, we have identified now a further 
NADPH-dependent L-xylulose reductase in $T$. reesei, which belongs, like the other two, to the group of short-chain dehydrogenase (Kallberg et al. 2002). This gene is not directly phylogenetically related to $A$. niger LxrA, but its deletion results in reduced growth on L-arabinose and Larabinitol (our unpublished data).

A xylitol dehydrogenase was first characterized in $T$. reesei, and it represents the first common enzyme of the shared pathway between L-arabinose and D-xylose catabolism. It is, therefore, both $\mathrm{D}$-xylose and L-arabinose inducible, and its deletion leads to a reduced growth rate on D-xylose, whereas growth rates on xylitol remained almost unaltered due to the expression of lad1 in a $\Delta x d h 1$ strain (Seiboth et al. 2003). The effect on growth rate differs also for L-arabinose and L-arabinitol. On L-arabinose, growth of the $\Delta x d h 1$ strain is not seriously affected, while strongly reduced on L-arabinitol. One of the reasons may be the general lower growth rate of $T$. reesei on Larabinose compared to L-arabinitol (Akel et al. 2009) and that its loss might be, therefore, better be compensated for by LAD1. Deletion of both lad1 and $x d h 1$ leads to the inability of $T$. reese $i$ to grow on L-arabinose or D-xylose and its corresponding polyols at all.

Phosphorylation of D-xylulose is catalyzed by a single enzyme as described for $A$. niger. The xylulokinase is essential for L-arabinose and D-xylose catabolism, and the encoding gene $A$. niger xkiA was cloned by complementation of a xylulokinase mutant due to its ability to regain growth on D-xylose (vanKuyk et al. 2001).

Beside the classical pathway, where D-xylulose 5phosphate is further assimilated in the pentose phosphate pathway, analysis of the genomes of several fungi revealed that some contain a putative phosphoketolase (EC 4.1.2.9) gene. This would raise the (theoretical) possibility for a shortcut catabolism of D-xylulose-5-phosphate to glyceraldehyde-3-phosphate and acetylphosphate, bypassing the pentose phosphate pathway (Flipphi et al. 2009), although respective enzyme activities could not be detected in A. niger cell-free extracts (Witteveen et al. 1989).

\section{Transcriptional regulation of L-arabinose degradation}

Expression of the extra- and intracellular enzymes is specifically and coordinately induced in response to the presence of L-arabinose or the downstream pathway intermediate L-arabinitol. Expression is usually repressed during growth on more readily utilizable monosaccharides, such as D-glucose even in the simultaneous presence of Larabinose, which can result from inducer exclusion or carbon catabolite repression. Since the accumulation of intracellular L-arabinitol correlates with higher production of the enzymes involved in arabinan breakdown in $A$. nidulans and $A$. niger, L-arabinitol is often regarded as the true inducer of the arabinanolytic system (de Vries 2003). Regulatory $A$. niger mutants of the L-arabinose pathway were described by (De Groot et al. 2003) and showed a decrease in both arabinanases and intracellular pathway gene expression in the presence of L-arabinose and Larabinitol, but the genes corresponding to these loci have not been cloned yet. The Zn(II)2-Cys6 binuclear cluster protein $X \ln R$ is a transcriptional activator of cellulase and xylanase gene transcription in different Aspergillus spp. and an orthologue, thereof, was also described for $T$. reesei (for a review see (Stricker et al. 2008)). However, XlnR has been reported not to regulate arabinan and L-arabinose metabolism in Aspergilli (de Groot et al. 2003). Recently the specific L-arabinose regulator AraR was identified, which controls the L-arabinose pathway and the extracellular degrading arabinanases (Battaglia et al. 2010). It also interacts with $X \ln R$ in the regulation of specific steps of the pentose catabolic pathway. Therefore, two positive regulators are involved in the pentose catabolic pathway of Larabinose and D-xylose in $A$. niger, which respond to either D-xylose $(\mathrm{X} \ln \mathrm{R})$ or L-arabinose/L-arabinitol (AraR), or both. AraR is able to compensate for loss of XlnR on Dxylose, whereas XlnR is hardly able to compensate for loss of AraR on L-arabinose. AraR seems to be the result of a gene duplication, which occurred specifically in the Eurotiales and is, therefore, not present in T. reesei. In analogy to $A$. niger, it was reported that in $T$. reesei, a knockout of the $x \ln R$ orthologue $x y r l$ did not affect growth of the fungus on L-arabinose and the total L-arabinose reductase activity (Stricker et al. 2006). However, a xyll gene knockout resulted in a strongly impaired growth on Larabinose due to the major involvement of XYL1 in the total L-arabinose reductase activity (Seiboth et al. 2007), and despite earlier claims, we found that the deletion of XYR1 leads to a decreased growth on L-arabinose. XYR1 regulates, however, only the first step of L-arabinose catabolism, since $\Delta x y r 1$ strains constitutively, expressing XYL1 can regain wild-type growth, and the growth rate of a $\Delta x y r 1$ strain on L-arabinitol is similar to the parental strain. Therefore, a specific transcriptional activator of the genes involved in the regulation of the catabolic pathway genes downstream of xyll in $T$. reesei and a regulator outside the Eurotiales for Larabinose catabolism is still not known.

In T. reesei, all four ABF-encoding genes (i.e., abfl-3; and $b x l 1)$ are L-arabinose and L-arabinitol inducible. Analysis of the influence of the early catabolic steps on expression of the ABF genes showed that loss of lad1 leads (similar to $A$. niger) to a strongly enhanced expression of all four $\mathrm{ABF}$-encoding genes, whereas their expression was severely impaired in an $\Delta x y l l$ strain. This impairment was also found on L-arabinitol, although XYL1 is not needed for growth on L-arabinitol. Deletion of the (hemi) cellulase 
regulator xyrl led to a loss of expression of two ABFencoding genes ( $a b f 2$ and $b x l 1)$, whereas the expression of $a b f 1$ and 3 was only slightly affected. The upregulation of the $a b f 1-3$ and bxl1 in the $\Delta l a d 1$ strain would be consistent with the hypothesis that L-arabinitol, which accumulates in the $\Delta l a d 1$ strain, is the intracellular inducer. However, a possible role of L-arabinose as inducer cannot be excluded, since Larabinose can be formed by the reverse reaction of XYL1 during growth on L-arabinitol. Respective L-arabinose levels were decreased in the $\Delta x y l 1$ strain but elevated in the $\Delta l a d l$ strain. In conclusion, the expression of the arabinanolytic genes in $T$. reesei seems to be regulated by an early pathway intermediate (L-arabinose or L-arabinitol), the first enzyme of the pathway XYL1, and in the case of abf2 and bxl1 the (hemi) cellulase regulator XYR1.

An interesting finding is that in $A$. niger, a number of Larabinose pathway genes (e.g., ladA, larA) are initially upregulated after transfer to D-xylose (Mojzita et al. 2010b). This finding might be an artifact, since D-xylose can contain small amounts of L-arabinose (Andersen et al. 2008). Considering that both D-xylose and L-arabinose are often concomitantly present in the plant cell wall polymers, such a coinduction could make sense for a saprobic fungus to effectively release and assimilate both pentoses. In this context, it is interesting that an overexpression of the Aspergillus oryzae $x \ln R$ led to an upregulation of genes involved in L-arabinose metabolism, including ladA and the lxrA orthologue (Noguchi et al. 2009).

\section{Pentose catabolic enzymes involved in D-galactose catabolism}

In eukaryotes, the hexose D-galactose is usually assimilated via the Leloir pathway (for a recent review: Sellick et al. 2008). The galactokinase of the Leloir pathway is specific for $\alpha$-D-galactose, and therefore, $\beta$-D-galactose has to be epimerized to its $\alpha$-anomer by a mutarotase (aldose 1 epimerase, EC 5.1.3.3) before it can be phosphorylated. Fungi, such as $A$. nidulans and $T$. reesei, have at least a second possibility at hand to utilize D-galactose, which was discovered by blocking of the Leloir pathway (Seiboth et al. 2004; Roberts 1970). In A. nidulans, this pathway was only active at $\mathrm{pH}>7$, and Roberts speculated that the pathway might start with an oxidative step by a D-galactose oxidase. Later it was shown that this pH-dependent growth was found only in the presence of ammonium but not nitrate as nitrogen source (Fekete et al. 2004). A nonphosphorolytic pathway has been suggested to be operative in, e.g., $A$. niger (Elshafei and Abdel-Fatah 2001), but its importance for D-galactose will need further investigations.

In $T$. reesei, the first identified pathway intermediate of this second D-galactose pathway was galactitol, which was discovered due to its transient accumulation during growth on D-galactose or lactose in a galactokinase deletion strain (Seiboth et al. 2004). In T. reesei, the catabolic path is initiated by the D-xylose reductase XYL1 (EC 1.1.1.21, Seiboth et al. 2007), which catalyzes the NADPHdependent reduction of D-galactose (beside D-xylose and L-arabinose) to galactitol. Using our collection of specific gene deletion strains in the L-arabinose and D-xylose pathway and testing these for their growth on the first pathway intermediate galactitol, further steps of the pathway could be identified (Fig. 3). The second step in this pathway, i.e., the $\mathrm{NAD}^{+}$dependent oxidation of galactitol is catalyzed by the $T$. reesei L-arabinitol 4dehydrogenase LAD1. $\Delta$ lad1 strains are unable to grow on galactitol, and a simultaneous block of the Leloir pathway at the galactosekinase step $(\Delta l a d 1 \Delta$ gall) led to the inability to grow on D-galactose. Characterization of the recombinant produced LAD1 led to the unexpected finding that galactitol is oxidized to L-xylo-3-hexulose (Pail et al.
Fig. 3 A second oxidoreductive pathway of D-galactose utilization. A second pathway for Dgalactose uilization was recently identified in $T$. reesei and $A$. nidulans. It starts with the reduction of D-galactose to galactitol by the D-xylose reductase XYL1 (aldose reductase) in $T$. reesei. A hypothetical draft of the pathway for the further degradation of galactitol is summarized

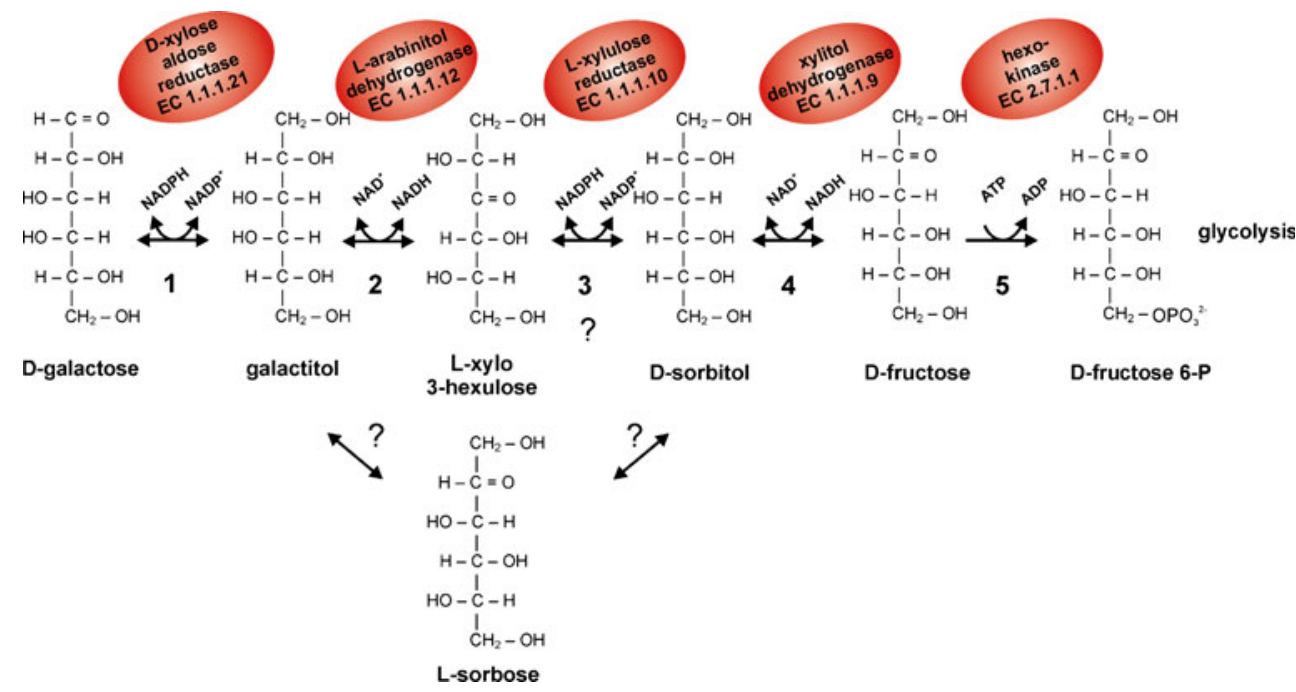


2004). This intermediate has not yet been identified in other fungi, and the further fate of this metabolite is currently under investigation. In analogy to the L-arabinose pathway, we hypothesize that an L-xylulose reductase might be responsible for its further catabolism. However, knockout strains in the $T$. reesei lxrl or a double deletion strain $(\Delta$ gall $\Delta l x r 1)$ grew indistinguishable from the parental strains QM9414 or the single deletion strain $\Delta$ gall on Dgalactose and galactitol (unpublished results). An obvious explanation for this finding is that LXR1-as described above-turned out to be also not involved in L-arabinose catabolism. Therefore, further research will concentrate on the $T$. reesei orthologue of the true L-xylulose reductase LxrA of A. niger in the catabolism of L-xylo-3-hexulose. In $A$. nidulans, L-sorbose was found to accumulate during growth on D-galactose but was absent in strains with a lossof-activity of L-arabinitol dehydrogenase (araA1), which suggests that L-sorbose is an intermediate of this Dgalactose pathway in A. nidulans (Fekete et al. 2004). Further L-sorbose catabolism involved a hexokinase step, indicated by the inability of the A. nidulans frA1 (fructose non-phosphorylating) mutant to grow on galactitol or Lsorbose and that an additional mutation in the galactokinase led to strains unable to grow on D-galactose. The role of hexokinase would imply that the metabolism of L-sorbose proceeds by reduction to D-sorbitol and subsequent oxidation to D-fructose. The L-sorbose step requires a reductase activity, which in analogy to the L-arabinose pathway, could be fulfilled by an L-xylulose reductase or similar enzyme.

From our data, it is however, not clear if the product of LAD1 L-xylo-3-hexulose has to be further converted to Lsorbose before it can be reduced to D-sorbitol. Further efforts will be necessary to elucidate this part of the pathway and to establish if there might be differences between $A$. nidulans and $T$. reesei. In T. reesei, D-sorbitol oxidation to D-fructose can be carried out by the T. reesei XDH1 (and partially also by LAD1), and in analogy to the pentose catabolic pathways, this D-galactose pathway ends with a phosphorylation of D-fructose to D-fructose 6phosphate, which is consistent with our results obtained with a hexokinase deletion strain of $T$. reesei, which is unable to grow on galactitol and D-sorbitol (Guangtao et al. 2010, our unpublished results).

The physiological relevance of this pathway for Dgalactose utilization seems to differ between $A$. nidulans and $T$. reesei. In $A$. nidulans, the pathway can fully compensate for the loss of the Leloir pathway (Roberts 1970; Fekete et al. 2004), while in T. reesei, inactivation of the Leloir pathway leads already to strains, which are significantly impaired in their growth on D-galactose. However, in $T$. reesei, this pathway is of importance for galactitol and lactose utilization and cellulase induction (Kubicek et al. 2009).

\section{Future perspectives}

Within the last years, our understanding of the arabinanases and L-arabinose catabolism has substantially increased, but a number of open questions remain in context with Larabinose uptake and the regulation of the arabinanolytic system outside of the Eurotiales. Further research will also be necessary to understand the L-xylulose reductase step in fungi and its implication for the redox factor balance of this pathway.

Acknowledgments BS and BM were supported by a grant of the Austrian Science Foundation (P19421).

Open Access This article is distributed under the terms of the Creative Commons Attribution Noncommercial License which permits any noncommercial use, distribution, and reproduction in any medium, provided the original author(s) and source are credited.

\section{References}

Akel E, Metz B, Seiboth B, Kubicek CP (2009) Molecular regulation of arabinan and L-arabinose metabolism in Hypocrea jecorina (Trichoderma reesei). Eukaryot Cell 8(12):1837-1844. doi:10.1128/EC.00162-09

Andersen MR, Vongsangnak W, Panagiotou G, Salazar MP, Lehmann L, Nielsen J (2008) A trispecies Aspergillus microarray: comparative transcriptomics of three Aspergillus species. Proc Natl Acad Sci USA 105(11):4387-4392

Battaglia E, Visser L, Nijssen A, van Veluw GJ, Wösten HAB, de Vries RP (2010) Analysis of regulation of pentose utilization in Aspergillus niger reveals evolutionary adaptations in the Eurotiales. Stud Mycol: in press

Beldman G, Schols HA, Pitson SM, Searle-van Leeuwen MJF, Voragen AGJ (1997) Arabinans and arabinan degrading enzymes. In: Sturgeon RJ (ed) Advances in Macromolecular Carbohydrate Research, vol 1. JAI Press Inc., London, pp 164

Cantarel BL, Coutinho PM, Rancurel C, Bernard T, Lombard V, Henrissat B (2009) The Carbohydrate-Active EnZymes database (CAZy): an expert resource for Glycogenomics. Nucleic Acids Res 37:D233-D238

Carapito R, Imberty A, Jeltsch JM, Byrns SC, Tam PH, Lowary TL, Varrot A, Phalip V (2009) Molecular basis of arabinobiohydrolase activity in phytopathogenic fungi: crystal structure and catalytic mechanism of Fusarium graminearum GH93 exoalpha-L-arabinanase. J Biol Chem 284(18):12285-12296

Chiang C, Knight SG (1961) L-Arabinose metabolism by cell-free extracts of Penicillium chrysogenum. Biochim Biophys Acta 46:271-278

Dahms AS, Anderson RL (1969) 2-keto-3-deoxyl-L-arabonate aldolase and its role in a new pathway of L-arabinose degradation. Biochem Biophys Res Commun 36(5):809-814

de Groot MJ, Prathumpai W, Visser J, Ruijter GJ (2005) Metabolic control analysis of Aspergillus niger L-arabinose catabolism. Biotechnol Prog 21(6):1610-1616

de Groot MJ, van de Vondervoort PJ, de Vries RP, vanKuyk PA, Ruijter GJ, Visser J (2003) Isolation and characterization of two specific regulatory Aspergillus niger mutants shows antagonistic regulation of arabinan and xylan metabolism. Microbiology 149 (Pt 5):1183-1191 
de Vries RP (2003) Regulation of Aspergillus genes encoding plant cell wall polysaccharide-degrading enzymes; relevance for industrial production. Appl Microbiol Biotechnol 61(1):10-20

de Vries RP, Visser J (2001) Aspergillus enzymes involved in degradation of plant cell wall polysaccharides. Microbiol Mol Biol Rev 65(4):497-522

de Vries RP, Flipphi MJ, Witteveen CF, Visser J (1994) Characterization of an Aspergillus nidulans L-arabitol dehydrogenase mutant. FEMS Microbiol Lett 123(1-2):83-90

Elshafei AM, Abdel-Fatah OM (2001) Evidence for a nonphosphorylated route of galactose breakdown in cell-free extracts of Aspergillus niger. Enzyme Microb Technol 29(1):76-83

Fekete E, Karaffa L, Sandor E, Banyai I, Seiboth B, Gyemant G, Sepsi A, Szentirmai A, Kubicek CP (2004) The alternative D-galactose degrading pathway of Aspergillus nidulans proceeds via L-sorbose. Arch Microbiol 181(1):35-44. doi:10.1007/s00203-003-0622-8

Flipphi M, Sun J, Robellet X, Karaffa L, Fekete E, Zeng AP, Kubicek CP (2009) Biodiversity and evolution of primary carbon metabolism in Aspergillus nidulans and other Aspergillus spp. Fungal Genet Biol 46(Suppl 1):S19-S44

Flipphi MJ, Panneman H, van der Veen P, Visser J, de Graaff LH (1993) Molecular cloning, expression and structure of the endo1,5-alpha-L-arabinase gene of Aspergillus niger. Appl Microbiol Biotechnol 40(2-3):318-326

Gielkens MM, Visser J, de Graaff LH (1997) Arabinoxylan degradation by fungi: characterization of the arabinoxylanarabinofuranohydrolase encoding genes from Aspergillus niger and Aspergillus tubingensis. Curr Genet 31(1):22-29

Guais O, Tourrasse O, Dourdoigne M, Parrou JL, Francois JM (2010) Characterization of the family GH54 alpha-L-arabinofuranosidases in Penicillium funiculosum, including a novel protein bearing a cellulose-binding domain. Appl Microbiol Biotechnol 87(3):1007-1021

Guangtao Z, Seiboth B, Wen C, Yaohua Z, Xian L, Wang T (2010) A novel carbon source-dependent genetic transformation system for the versatile cell factory Hypocrea jecorina (anamorph Trichoderma reesei). FEMS Microbiol Lett 303(1):26-32

Hahn-Hägerdal B, Karhumaa K, Jeppsson M, Gorwa-Grausl MF (2007) Metabolic engineering for pentose utilization in Saccharomyces cerevisiae. Adv Biochem Engin 108:147-177. doi: 10.1007/10 2007062

Hayn M, Steiner W, Klinger R, Steinmüller H, Sinner M, Esterbauer H (1993) Bioconversion of forest and agricultural plant residues. CAB International, Wallingford, UK

Herrmann MC, Vrsanska M, Jurickova M, Hirsch J, Biely P, Kubicek CP (1997) The beta-Dxylosidase of Trichoderma reesei is a multifunctional beta-D-xylan xylohydrolase. Biochem J. 321 (Pt 2):375-381

Kallberg Y, Oppermann U, Jornvall H, Persson B (2002) Short-chain dehydrogenase/reductase (SDR) relationships: a large family with eight clusters common to human, animal, and plant genomes. Protein Sci 11(3):636-641

Kimura I, Yoshioka N, Kimura Y, Tajima S (2000) Cloning, sequencing and expression of an alpha-L-arabinofuranosidase from Aspergillus sojae. J Biosci Bioeng 89(3):262-266

Kubicek CP, Mikus M, Schuster A, Schmoll M, Seiboth B (2009) Metabolic engineering strategies for the improvement of cellulase production by Hypocrea jecorina. Biotechnol Biofuels 2:19

Margolles-Clark E, Tenkanen M, Nakari-Setala T, Penttilä M (1996) Cloning of genes encoding alpha-L-arabinofuranosidase and beta-xylosidase from Trichoderma reesei by expression in Saccharomyces cerevisiae. Appl Environ Microbiol 62(10):38403846

Metz B, de Vries RP, Polak S, Seidl V, Seiboth B (2009) The Hypocrea jecorina (syn. Trichoderma reesei) lxrl gene encodes a D-mannitol dehydrogenase and is not involved in L-arabinose catabolism. FEBS Lett 583(8):1309-1313. doi:10.1016/j.febslet. 2009.03.027

Miyanaga A, Koseki T, Miwa Y, Mese Y, Nakamura S, Kuno A, Hirabayashi J, Matsuzawa H, Wakagi T, Shoun H, Fushinobu S (2006) The family 42 carbohydrate-binding module of family 54 alpha-L-arabinofuranosidase specifically binds the arabinofuranose side chain of hemicellulose. Biochem J 399(3):503-511

Mojzita D, Penttilä M, Richard P (2010a) Identification of an Larabinose reductase gene in Aspergillus niger and its role in Larabinose catabolism. J Biol Chem 285(31):23622-23628

Mojzita D, Vuoristo K, Koivistoinen OM, Penttilä M, Richard P (2010b) The 'true' L-xylulose reductase of filamentous fungi identified in Aspergillus niger. FEBS Lett 584(16):3540-3544

Noguchi Y, Sano M, Kanamaru K, Ko T, Takeuchi M, Kato M, Kobayashi T (2009) Genes regulated by AoXlnR, the xylanolytic and cellulolytic transcriptional regulator, in Aspergillus oryzae. Appl Microbiol Biotechnol 85(1):141-154

Numan MT, Bhosle NB (2006) Alpha-L-arabinofuranosidases: the potential applications in biotechnology. J Ind Microbiol Biotechnol 33(4):247-260

Pail M, Peterbauer T, Seiboth B, Hametner C, Druzhinina I, Kubicek CP (2004) The metabolic role and evolution of L-arabinitol 4dehydrogenase of Hypocrea jecorina. Eur J Biochem 271 (10):1864-1872. doi:10.1111/j.1432-1033.2004.04088.x

Richard P, Londesborough J, Putkonen M, Kalkkinen N, Penttilä M (2001) Cloning and expression of a fungal L-arabinitol 4dehydrogenase gene. J Biol Chem 276(44):40631-40637

Richard P, Putkonen M, Vaananen R, Londesborough J, Penttilä M (2002) The missing link in the fungal L-arabinose catabolic pathway, identification of the L-xylulose reductase gene. Biochemistry 41(20):6432-6437

Roberts CF (1970) Enzyme lesions in galactose non-utilising mutants of Aspergillus nidulans. Biochim Biophys Acta 201(2):267-283

Rye CS, Withers SG (2000) Glycosidase mechanisms. Curr Opin Chem Biol 4(5):573-580

Saha BC (2000) Alpha-L-arabinofuranosidases: biochemistry, molecular biology and application in biotechnology. Biotechnol Adv 18(5):403-423

Schleif R (2000) Regulation of the L-arabinose operon of Escherichia coli. Trends Genet 16(12):559-565

Seiboth B, Gamauf C, Pail M, Hartl L, Kubicek CP (2007) The D-xylose reductase of Hypocrea jecorina is the major aldose reductase in pentose and D-galactose catabolism and necessary for betagalactosidase and cellulase induction by lactose. Mol Microbiol 66(4):890-900. doi:10.1111/j.1365-2958.2007.05953.x

Seiboth B, Hartl L, Pail M, Fekete E, Karaffa L, Kubicek CP (2004) The galactokinase of Hypocrea jecorina is essential for cellulase induction by lactose but dispensable for growth on D-galactose. Mol Microbiol 51(4):1015-1025

Seiboth B, Hartl L, Pail M, Kubicek CP (2003) D-xylose metabolism in Hypocrea jecorina: loss of the xylitol dehydrogenase step can be partially compensated for by lad1-encoded L-arabinitol-4dehydrogenase. Eukaryot Cell 2(5):867-875

Sellick CA, Campbell RN, Reece RJ (2008) Galactose metabolism in yeast-structure and regulation of the leloir pathway enzymes and the genes encoding them. Int Rev Cell Mol Biol 269:111-150

Shallom D, Belakhov V, Solomon D, Gilead-Gropper S, Baasov T, Shoham G, Shoham Y (2002) The identification of the acid-base catalyst of alpha-arabinofuranosidase from Geobacillus stearothermophilus T-6, a family 51 glycoside hydrolase. FEBS Lett 514(2-3):163-167

Shallom D, Shoham Y (2003) Microbial hemicellulases. Curr Opin Microbiol 6(3):219-228

Skjot M, Kauppinen S, Kofod LV, Fuglsang C, Pauly M, Dalboge H, Andersen LN (2001) Functional cloning of an endo-arabinanase from Aspergillus aculeatus and its heterologous expression in A. oryzae and tobacco. Mol Genet Genomics 265(5):913-921 
Stricker AR, Grosstessner-Hain K, Wurleitner E, Mach RL (2006) Xyr1 (xylanase regulator 1) regulates both the hydrolytic enzyme system and D-xylose metabolism in Hypocrea jecorina. Eukaryot Cell 5(12):2128-2137

Stricker AR, Mach RL, de Graaff LH (2008) Regulation of transcription of cellulases- and hemicellulases-encoding genes in Aspergillus niger and Hypocrea jecorina (Trichoderma reesei). Appl Microbiol Biotechnol 78(2):211-220

vanKuyk PA, de Groot MJ, Ruijter GJ, de Vries RP, Visser J (2001) The Aspergillus niger D-xylulose kinase gene is co-expressed with genes encoding arabinan degrading enzymes, and is essential for growth on D-xylose and L-arabinose. Eur J Biochem 268(20):5414-5423

van Peij NN, Brinkmann J, Vrsanská M, Visser J, de Graaff LH (1997) beta-Xylosidase activity, encoded by $x \ln \mathrm{D}$, is essential for complete hydrolysis of xylan by Aspergillus niger but not for induction of the xylanolytic enzyme spectrum. Eur J Biochem 245(1):164-173

Verho R, Putkonen M, Londesborough J, Penttilä M, Richard P (2004) A novel NADH-linked L-xylulose reductase in the
L-arabinose catabolic pathway of yeast. J Biol Chem 279 (15):14746-14751

Watanabe S, Shimada N, Tajima K, Kodaki T, Makino K (2006) Identification and characterization of L-arabonate dehydratase, L2-keto-3-deoxyarabonate dehydratase, and L-arabinolactonase involved in an alternative pathway of L-arabinose metabolism. Novel evolutionary insight into sugar metabolism. J Biol Chem 281(44):33521-33536

Wenger JW, Schwartz K, Sherlock G (2010) Bulk segregant analysis by high-throughput sequencing reveals a novel xylose utilization gene from Saccharomyces cerevisiae. PLoS Genetics 6(5): e1000942

Witteveen CFB, Busink R, van de Vondervoort P, Dijkema C, Swart K, Visser J (1989) L-Arabinose and D-xylose catabolism in Aspergillus niger. J Gen Microbiol 135:2163-2171

Zechel DL, Withers SG (2000) Glycosidase mechanisms: anatomy of a finely tuned catalyst. Acc Chem Res 33(1):11-18

Zykwinska AW, Ralet MC, Garnier CD, Thibault JF (2005) Evidence for in vitro binding of pectin side chains to cellulose. Plant Physiol 139(1):397-407 